\title{
Criticality of Schedule Constraints - Classification and Identification for Project Management
}

\author{
Qui T. Nguyen ${ }^{1}$ and David K. H. Chua ${ }^{2}$ \\ ${ }^{1} \mathrm{PhD}$ Candidate, Department of Civil and Environmental Engineering, National University of Singapore, \\ 10, Kent Ridge Crescent, 119260, Singapore, E-mail: ntqui@nus.edu.sg (corresponding author). \\ ${ }^{2}$ Associate Professor, Department of Civil Engineering, National University of Singapore, \\ 10, Kent Ridge Crescent, 119260, Singapore, E-mail: ceedavid@ nus.edu.sg \\ Project Management \\ Best Paper of EPPM 2012 Conference \\ Received April 24, 2013; received revision May 29, 2013; accepted June 9, 2013 \\ Available online November 12, 2013
}

\begin{abstract}
In construction scheduling, constraints among activities are vital as they govern the schedule solution. Understanding their criticality is essential for better schedule management. This paper presents a systematic method to classify and identify the criticality of schedule constraints for the schedule management from the constraint perspective. In terms of criticality, schedule constraints can be grouped into four types: project-critical, activity-critical, sequencecritical and non-critical. Project-critical constraints are those which govern start/finish time of critical activities and the project end time. Activity-critical constraints define the start/finish time of non-critical activities, and sequence-critical constraints are those whose existence affect the start/finish time of some activities or the project end time. Constraints belonging to any of these groups are vital to a schedule as they cannot be removed from the constraint collection. Noncritical constraints, on the other hand, do not govern either start/finish time of any activity or the project end time. Accordingly, non-critical constraints are redundant and can be removed from the constraint collection without causing any change to the schedule solution. The method proposed was applied to a illustrative case example based on the construction of the main entrance of a nursing house for further interpretation. The proposed classification scheme could shed light on a more in-depth understanding of the nature of criticality and the role of constraints in a schedule, and thus better schedule management may be achieved.
\end{abstract}

Keywords: Constraint criticality, constraint management, schedule management

\section{Instruction}

In a construction project, schedule constraints represent the project requirements that a schedule must satisfy. They define the precedence relationships among activities as well as the sequences that construction processes may follow (Yeoh, 2012). Each constraint exhibits different influence to the schedule according to its characteristic and/or the activities involved. A constraint could be of no significance, locally significant to an activity or globally crucial for the entire schedule. In some cases, a constraint, when exists, have no impact on the schedule, yet removing it from the constraint collection could lead to changes in activity sequence for a better project makespan.

Constraint management is an essential task of schedule management. The major aim is to identify and prioritize the "key" or critical constraints that govern the overall schedule (Rahman, 1998). Generally, a critical constraint could be any that control the project duration, the start and/or finish times of an activity or the sequence currently defined in the schedule. In other words, a critical constraint cannot be removed from the constraint collection, since such a removal will lead to changes in the schedule. Accordingly, the definition of critical constraints is likely broader than that of critical activities. While critical activities are those explicitly shown in the critical path(s), a critical constraint could be between two noncritical activities. It also means that there could be different types of criticality dependent on how a constraint affects a certain activity or the overall schedule. Thus, understanding and classifying the nature of constraint criticality is necessary for constraint management as well as schedule controlling.

The concept of criticality already has been introduced since the formation of the Critical Path Method (CPM) (Kelley, 1961) in the 1950's. CPM allows planners to identify critical paths and critical activities, from which critical constraints can be implicitly inferred as those connecting two critical activities. In order to provide an insight to the criticality of an activity, previous researchers have proposed different classification schemas, including Wiest (1981), Moder et al. (1983), or Valls and Lino (2001). However, as CPM has limitations in representing non-precedence constraints such as work/resource 
continuity or process concurrency/ overlap/ disjunction (El-Bibany, 1997; El-Rayes and Moselhi, 2001), inferring critical constraints from critical paths could generally be inadequate.

Developing criticality concept in resource-constrained scheduling problems has received much research attention, such as (Wiest, 1981; Woodworth and Shanahan, 1988; Bowers, 1995; Lu and Li, 2003; Rivera and Duran, 2004). Their major focus is to identify resource-constrained critical paths. Critical constraints, either precedence or resource constraints, could also be determined from critical paths as they are the constraints connecting critical activities. The idea of critical constraints is therefore still restricted to those that affect the final project end time, and constraints among non-critical activities could be intuitively considered non-critical.

Schedule constraints in a construction project can be of any types, not only precedence or resource constraints. They impose the conditions that an activity can start, process and finish. In this context, a critical constraint may not necessarily be between two critical activities. Chua and Shen (2005) proposed a methodology to identify key information and resource constraints in a delayed project. In their model, information and resource constraints are modeled as unary temporal constraints of activities, and the impact of constraints to the overall project performance is measured using constraint float. This method helps planner highlight the hidden bottleneck constraints so that appropriate policies can be utilized to lessen the delay.

According to the above review, although many methodologies have been proposed to develop the concept of criticality in construction schedules, it is found that there is still room for improvement. Firstly, critical constraints could not be restricted to those between critical activities. From the construction viewpoint, not only the project duration but also the start/ finish times of all activities are of importance to contractors, as they may affect their overall working plan among different projects. While each activity may be involved in different constraints, it is regular that only some of them actually control the activity's times. Thus, these constraints should also have higher priority for better management. Secondly, schedule constraints generally can be of any types, such as unary or binary, and with minimal-lag, maximal-lag or non-lag requirements. Thus, a generic and systematic approach which can be applied to all constraint types is necessary. Lastly, critical constraints should be identified as early as in the planning phase, so that better management strategies can be applied for better schedule performance. Since schedule is a critical success factor of construction project (Al-Otaibi et al., 2013), constraint analysis will thus improve the overall project performance.

This paper aims to investigate the criticality in construction schedules from a constraint viewpoint. It presents a systematic methodology for classifying and identifying critical schedule constraints. Both unary and binary with minimal-lag, maximal-lag and non-lag constraints are examined. In the context of this paper, schedule constraints are defined as temporal interval constraints and captured using the PDM++ model (Chua and Yeoh, 2011), which is briefly summarized in the next section. Then, a detailed description of the proposed methodology is presented, followed by the demonstration of its application via an illustrative example schedule.
Subsequently, a brief comparison between constraint criticality and activity criticality is presented to highlight the differences and the advantages of identifying constraint criticality. By categorizing and determining constraint criticality in a systematic way, the proposed methodology could help provide a deeper understanding about their role to the overall schedule, so that better schedule management could be achieved.

\section{Representing Temporal Constraints}

Schedule constraints are derived from construction requirements which represent all project's constraint and concerns to which construction processes and product facilities must conform for conducting procurement, construction, and logistic processes (Song and Chua, 2006). Schedule constraints can exist in different forms such as functional requirements, resource or safety constraints (Nguyen et al., 2009). For scheduling purpose, all constraints need to be eventually converted into temporal relationships between activities. The four types of precedence relationships (Finish-Start (FS), FinishFinish (FF), Start-Start (SS), and Start-Finish (SF)) used in $\mathrm{CPM} / \mathrm{PDM}$ are represented in a point-to-point format. In addition to simplicity, this representation format also provides mechanism to represent lag time requirements. However, point-to-point format is found to be inadequate in representing complex constraints such as work/resource continuity or disjunction, and process concurrency/ overlapping (Jaafari, 1984; El-Rayes and Moselhi, 2001). Moreover, the four relationships still do not consider maximal lag type constraints (Neumann and Zhan, 1995) which are also necessary for construction projects.

Interval-to-interval representation (Allen, 1984) contains a set of 13 binary temporal relations which completely depicts all possible relationships between two time intervals. This representation format is found to provide greater flexibility and a richer semantic context to explicitly describe the precedence, coincidence, concurrency, and disjunction constraints between two time intervals. Therefore, it overcomes the aforementioned limitations of the point-to-point format. Yet, from a scheduling viewpoint, while lag time requirements are common in construction schedule, Allen's relationships still lack a mechanism to capture these requirements.

The PDM++ model (Chua and Yeoh, 2011) which is adopted in this paper integrates the advantages of the aforementioned representation paradigms. It extends the traditional PDM model by incorporating two basic logical operators "AND" and "OR" with the enriched syntax inspired by the Artificial Intelligence developed by Allen (1984). Accordingly, PDM++ not only maintains the capability of Allen's representation but also subsumes the PDM model by allowing both minimum and maximum lag time requirements to be explicitly described. PDM++ generally comprises two different types of relationships: Unary and Binary. Unary relationships are defined as constraints influencing a single activity while binary relationships specify the temporal constraints between two activities. With a complete set of temporal relationships, PDM++ could be employed to model complex temporal constraints in construction schedules. For the ease of reading, a summarized description of these constraints is presented in Fig. 1, where $X^{-}$and $d_{X}$ respectively denote the start time and duration of activity $X$, and $m(m \geq 0)$ and 
$\sim m$ respectively denotes minimal and maximal lag requirement.

\begin{tabular}{|c|c|c|c|}
\hline Constraint & $\begin{array}{l}\text { Short } \\
\text { Form }\end{array}$ & $\begin{array}{c}\text { Mathematic } \\
\text { Definition }\end{array}$ & $\begin{array}{c}\text { Pictorial } \\
\text { Representation }\end{array}$ \\
\hline$X$ Start-Before $(m)$ & SB & $x^{-} \leq m$ & $m_{x}$ \\
\hline$X$ Start-After $(m)$ & SA & $x^{-} \geq m$ & \\
\hline$X$ Due-Before $(m)$ & DB & & \\
\hline$X$ Due-After $(m)$ & DA & $x^{-}+d_{X} \geq m$ & \\
\hline $\begin{array}{l}X \text { Before }(m) Y \\
X \text { Before }(\sim m) Y\end{array}$ & B & $\begin{array}{l}X^{-}+d_{X}+m \leq Y^{-} \\
X^{-}+d_{X}+m \geq Y^{-}\end{array}$ & \\
\hline $\begin{array}{l}X \text { Starts }(m) Y \\
X \text { Starts }(\sim m) Y\end{array}$ & SS & $\begin{array}{l}X^{-}+m \leq Y^{-} \\
X^{-}+m \geq Y^{-}\end{array}$ & \begin{tabular}{|l|}
$\mathrm{X}$ \\
$\mathrm{Y}$ \\
$\leftarrow \mathrm{m} \rightarrow$ \\
\end{tabular} \\
\hline $\begin{array}{l}X \text { Finishes }(m) Y \\
X \text { Finishes }(\sim m) Y\end{array}$ & $\mathrm{FF}$ & $\begin{array}{l}X^{-}+d_{X}+m \leq Y^{-}+d_{Y} \\
X^{-}+d_{X}+m \geq Y^{-}+d_{Y}\end{array}$ & \begin{tabular}{r}
\multicolumn{1}{c}{$\mathrm{X}$} \\
$\mathrm{Y}$ \\
\end{tabular} \\
\hline$X$ Start-Finish $(m) Y$ & & $X^{-}+m \leq Y^{-}+\alpha_{\gamma}$ & $\approx$ \\
\hline $\begin{array}{c}X \text { Start- } \\
\text { Finishes }(\sim m) Y\end{array}$ & SF & $X^{-}+m \geq Y^{-}+d_{Y}$ & \\
\hline$X$ Overlaps $(m) Y$ & 0 & $\begin{array}{c}\left(X^{-}+m \leq Y^{-}+d_{Y}\right) \wedge \\
\left(Y^{-}+m \leq X^{-}+d_{X}\right)\end{array}$ & $\frac{X}{\mathrm{X}}$ \\
\hline$X$ Overlaps $(\sim m) Y$ & 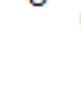 & $\begin{array}{c}\left(X^{-}+d_{X} \leq Y^{-}+m\right) \vee \\
\left(Y^{-}+d_{Y} \leq X^{-}+m\right)\end{array}$ & \\
\hline$X$ Meets $Y$ & M & $\begin{array}{c}\left(X+d_{X}+m \leq Y^{-}\right) \wedge \\
\left(X+d_{X}+m \geq Y^{-}\right)\end{array}$ & $\mathrm{x}$ \\
\hline$X$ Contains $Y$ & C & $\begin{array}{c}\left(X \geq Y^{-}\right) \wedge \\
\left(X+d_{X} \leq Y^{-}+d_{Y}\right)\end{array}$ & \begin{tabular}{|c|}
$\mathrm{X}$ \\
$\mathrm{Y}$ \\
\end{tabular} \\
\hline$X$ Disjoint $Y$ & D & $\begin{array}{c}\left(X^{-}+d_{X} \leq Y^{-}\right) Y \\
\left(Y^{-}+d_{Y} \leq X^{-}\right)\end{array}$ & 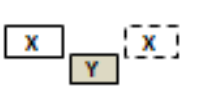 \\
\hline
\end{tabular}

Fig. 1. PDM++ temporal interval constraints

\section{Criticality of Schedule Constraints}

Constraints could have different impacts on the schedule, due to their nature and the activities involved. Thus, a classification of constraint criticality would be useful to further distinguish the significance of constraints to the schedule. A constraint is considered critical if its existence affects the project duration, activity's start/finish times or the sequence between activities. In other words, any change or removal of a critical constraint will lead to variations to the schedule. In contrast, non-critical constraints are redundant ones, and deleting such constraints results in no change to either schedule times or sequence. From this perspective, this paper categorizes constraint into four groups, termed as: project-critical, activity-critical, sequence-critical, and non-critical.

\subsection{Project-critical Constraint}

A constraint is project-critical when it governs the project duration. Since project duration is defined by start/finish times of critical activities, a project-critical constraint is the one that directly controls the start/finish times of a critical activity. By this definition, it is apparent that there is a correlation between a critical activity path and a project-critical constraint path. More precisely, any critical activity path has an associated project-critical constraint path, which passes through the constraints governing the start/finish times of the critical activities involved. As a result, project-critical constraints can be implicitly derived from critical activity paths.

For illustration, Fig. 2 depicts a simple schedule network with 7 activities and 13 constraints, with a total duration of 20 days. The modeling syntax of constraints follows the legend shown in Fig. 1. The critical activity path is A-C-D-E-G. This path also includes four projectcritical constraints named $c_{2}, c_{6}, c_{8}$, and $c_{13}$. These constraints directly define the start/finish times of activities $\mathrm{A}, \mathrm{C}, \mathrm{D}, \mathrm{E}$, and $\mathrm{G}$ respectively. When $c_{2}$ is modified to $B(1)$, start and finish times of activity $\mathrm{C}$ also change to new values as 6 and 10 respectively, resulting in a new project duration of 21 days.

\subsection{Activity-critical Constraint}

Similar to critical activities, the start/finish times of every non-critical activity are also controlled by at least one constraint. These times can be changed due to any change or deletion of such a constraint. Although it may not be critical to project duration, constraints of this type are also vital to the schedule. In addition, an activity-critical constraint becomes project-critical if the activities involved are critical. Alternatively, it is possible to state that project-critical constraint is a subclass of activitycritical constraint which defines a relationship between two critical activities. In the example schedule shown in Fig. 2, B is a non-critical activity and its start/finish times are controlled by two constraints $c_{1}$ and $c_{4}$. In detail, constraint $c_{1}$ defines its early start/finish times while constraint $c_{4}$ governs its late start/finish times. In the case that the constraint has some change, the controlled times are also affected, while the project duration is not be influenced. For example, if $c_{1}$ is changed to $S S(3)$, early start/finish times of $B$ will change to 1 and 11 respectively. Yet, the project duration remains at 20 days.

\subsection{Sequence-critical Constraint}

When a constraint does not control start/finish times if any activity, it is commonly considered "non-critical". Consequently, it is easily to be intuitively treated as a redundant constraint, which means that any change or removal of such a constraint is considered not to cause any change to the schedule. However, it possibly happens that when a non-critical constraint is deleted, the sequence among activities can also be changed to achieve a shorter project duration.

Constraint $c_{11}$ in Fig. 2 is an example of this situation. In the current schedule, activity $\mathrm{D}$ is scheduled before activity $\mathrm{E}$ as this sequence provides better project duration. (In the other sequence where D starts after E due to the disjoint constraint, the project duration is 22 days). $c_{11}$ can be considered non-critical as it does not control any activity's times. Yet, if it is omitted, the preferable sequence will switch to A-C-E-D-G, with shorter project duration of 18 days (as shown in Fig. 3). (If D starts before $\mathrm{E}$, project duration is unchanged at 20 days).

Under some other circumstances, removing a "noncritical" constraint may allow infeasible sequence become feasible. As illustrated in Fig. 4, with the existence of constraint $c_{4}$, there is only one feasible sequence in which 
activity $\mathrm{B}$ is before activity $\mathrm{C}$, giving the project makespan of 20 days (Fig. 4a). However, when $c_{4}$ is removed, the other sequence in which activity $C$ is before activity $B$ becomes feasible with a shorter project duration of 17 days (Fig. 4b) while the makespan of the original sequence remains unchanged. From these examples, there are some special constraints whose existence affects the activity sequence producing best project makespan. This class of constraints is defined as "sequence-critical" in this paper. Due to this distinctive characteristic, sequence-critical constraints thus could not be treated as redundant constraints.
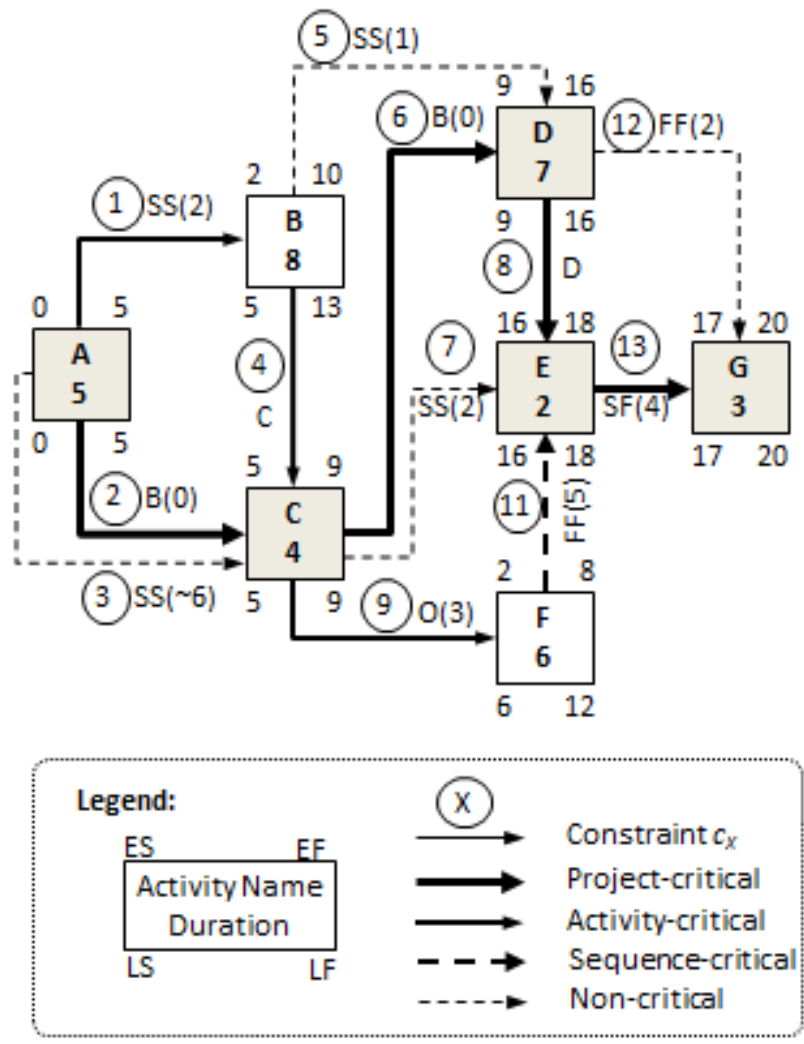

Fig. 2. Example schedule network

\subsection{Non-critical Constraint}

The last category of constraint is "non-critical". It refers to constraints which do not control the start/finish times of any activity. As such, removal of non-critical constraints will cause no change to the schedule. Hence, they could be considered redundant constraints.

\subsection{Order of Constraint Criticality}

From the above definitions, constraint criticality in a schedule can be ordered as follows:

\section{Project-critical $\geq$ Activity-critical $\geq$}

Sequence-critical $\geq$ Non-critical

Project-critical constraints are apparently the most crucial since not satisfying them can delay the entire project. Secondly, activity-critical constraints also need to be well-managed in order to maintain activity's times as planned. Failure to fulfill these constraints could result in float consumption. Although not crucial to the schedule times, sequence-critical constraints cannot be ignored since their removal can lead to project improvement.
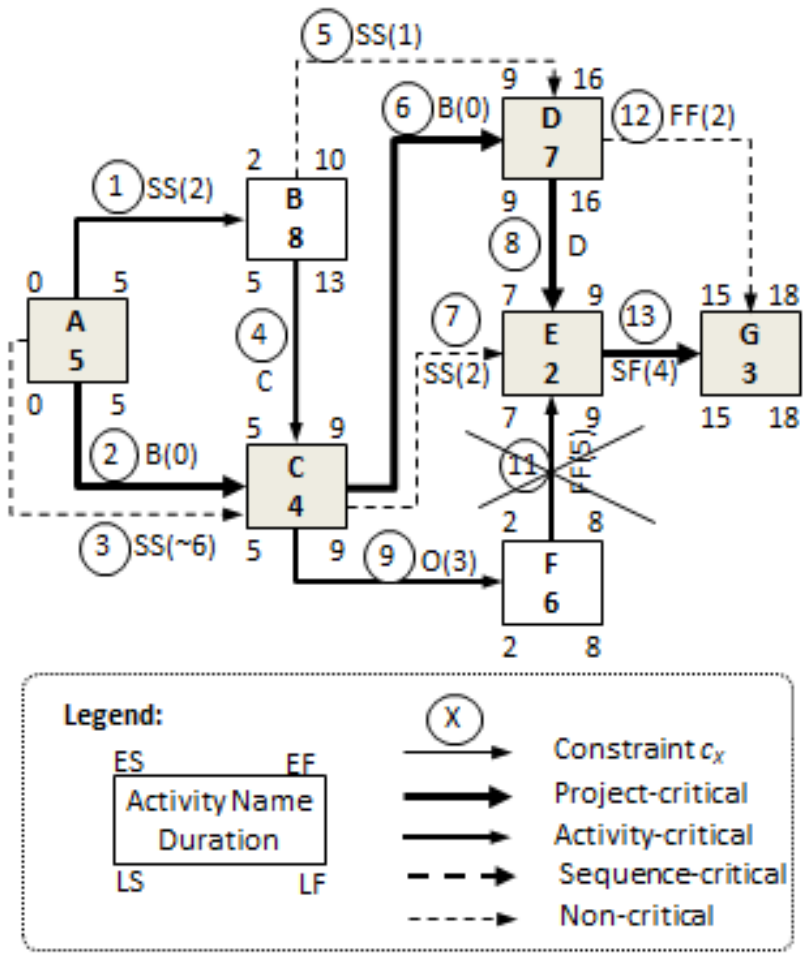

Fig. 3. Example schedule network - Removal of $c_{11}$

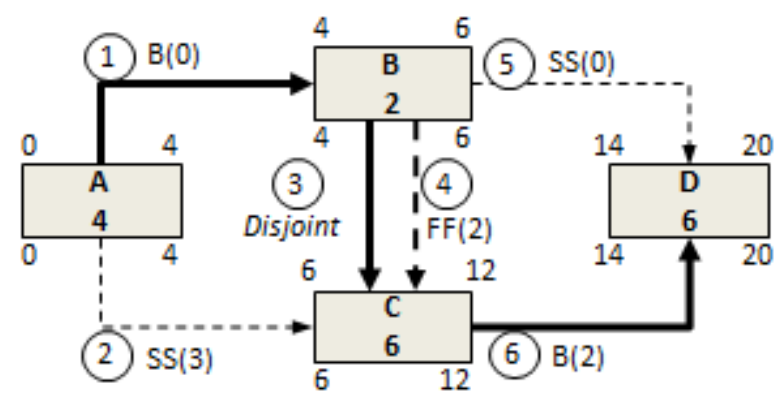

A-B-C-D: $T_{p}=20$ days A-C-B-D: Infeasible

(a) Existence of $c_{4}$

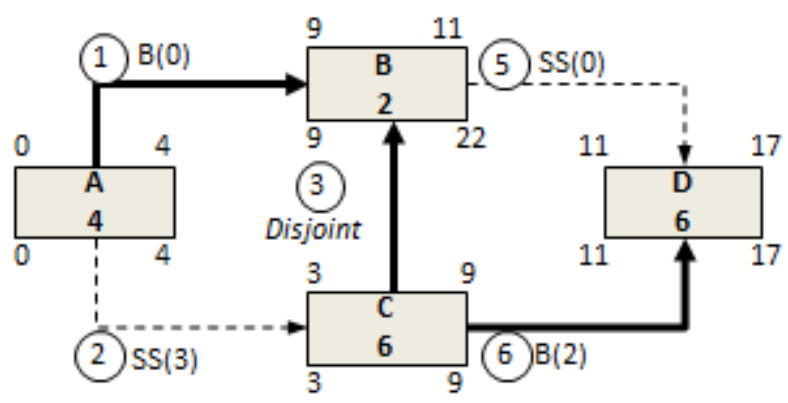

A-B-C-D: $T_{p}=20$ days A-C-B-D: $T_{p}=17$ days

(b) Removal of $c_{4}$

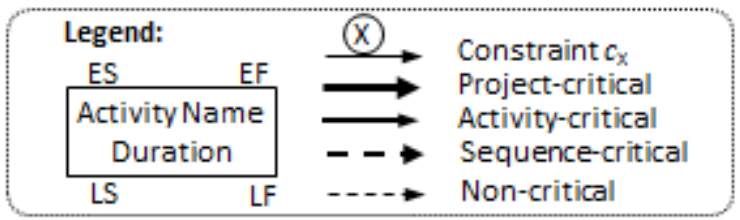

Fig. 4. Example of sequence-critical constraint 


\section{Identification of Constraint Criticality}

Identifying the criticality type of a constraint seems to be simple in small schedules with a small number of activities and constraints. However, as construction projects commonly involve tens or hundreds of activities and constraints, and manually checking each constraint for its criticality is clearly time-consuming and probably impossible. Therefore, a systematic methodology for identifying constraint criticality is obviously a necessity. For management purpose, a criticality indicator is also essential for constraint comparison and evaluation.

Similar to activity criticality, constraint criticality may be determined based on criticality indicators. The criticality of a constraint is closely related to whether it may prolong schedule makespan or may reduce the feasible ranges of activities' start times when the constraint becomes more obstructive to project performance or more tightened. In other words, if a constraint has less room for being tightened, it yields a higher degree of criticality. Accordingly, the present methodology utilizes the tightening degree, or tightening time, as an indicator of constraint criticality.

The tightening time of a constraint can be determined by examining how much it can adversely vary without causing changes to the activities involved. Alternatively, it can be determined by the introduction of flexibility measures of the activities involved. Alternatively, it can be determined by identifying how much an activity can be flexibly moved backward or forward without violating that particular constraint. The flexibility of an activity $X$ of a constraint $C$, denoted as $f_{X, C}$, is the minimal amount of time that $X$ can be moved forward/backward (called forward/backward flexibility times and denoted as $f_{k, C}^{F W}$ and $f_{k, C}^{B W}$, respectively), from the original time range without violating $C$, while its duration, the feasible time range of the other activity involved in $C$ and lag time remain unchanged, given by:

$$
f_{X, C}=\min \left(f_{k, C}^{F W}, f_{k, C}^{B W}\right)
$$

Let $k^{-}=\left[\begin{array}{lll}E S_{k} & . . L S_{k}\end{array}\right]$ denote the original feasible start time of activity k where $E S_{k}$ and $L S_{k}$ are early and late start time of $k$, and $k_{C}{ }^{-}=\left[E S_{k, C} \ldots L S_{k, C}\right]$ the start time range of $k$ defined only by constraint $c$. The forward/backward flexibility time of activity $k$ regarding constraint $C$ respectively can be determined from the difference between ${ }^{k_{C}^{-}}$and $k^{-}$as follows:

$$
\begin{aligned}
f_{k, C}^{F W} & =L S_{k, C}-E S_{k} \\
f_{k, C}^{B W} & =L S_{k}-E S_{k, C}
\end{aligned}
$$

A constraint cannot be further tightened when its involved activities cannot vary or have no flexibility. Thus, the tightening time of a constraint $C$ between two activities $X$ and $Y$ (denoted as $T_{C}$ ) is the minimal flexibility of $\mathrm{X}$ and $Y$, shown as:

$$
T_{C}=\min \left(f_{X, C}, f_{Y, C}\right)
$$

\begin{tabular}{|c|c|c|}
\hline $\begin{array}{l}\text { Criticality } \\
\text { type }\end{array}$ & $\begin{array}{c}\text { Impact when } \\
\text { varied or removed }\end{array}$ & Indicator \\
\hline $\begin{array}{l}\text { Project- } \\
\text { critical }\end{array}$ & $\begin{array}{l}\text { Change of critical } \\
\text { activities' times } \\
\text { and/or project } \\
\text { duration }\end{array}$ & $\begin{array}{l}\text { - Between critical } \\
\text { activities } \\
\text { - } \mathrm{TC}=0\end{array}$ \\
\hline $\begin{array}{l}\text { Activity- } \\
\text { critical }\end{array}$ & $\begin{array}{l}\text { Change of non- } \\
\text { critical activities' } \\
\text { times }\end{array}$ & $\begin{array}{l}\text { - Not project critical } \\
-\mathrm{TC}=0\end{array}$ \\
\hline $\begin{array}{l}\text { Sequence- } \\
\text { critical }\end{array}$ & $\begin{array}{l}\text { Change of } \\
\text { activities' times, } \\
\text { project times } \\
\text { and/or sequence } \\
\text { when removed }\end{array}$ & $\begin{array}{l}-\mathrm{TC}>0 \\
\text { - Better project } \\
\text { makespan obtained } \\
\text { when removed }\end{array}$ \\
\hline Non-critical & No impact & $\begin{array}{l}-\mathrm{TC}>0 \\
\text { - No change in } \\
\text { project makespan } \\
\text { when removed }\end{array}$ \\
\hline
\end{tabular}

Table 1. Indicator of constraint criticality

Tightening time represents how much a constraint can be varied without affecting an activity's times and/or project time. Thus, $T_{C}=0$ indicates that constraint $C$ cannot be tightened anymore, and thus it is either projectcritical (when linking two critical activities) or activitycritical otherwise. When $T_{C}>0$, constraint $C$ still have room for tightening and thus it is either sequence-critical or non-critical. In this case, the schedule needs to be further analyzed by re-computing it without the existence of $C$. If there no change in activity's times or sequence, $C$ is non-critical; otherwise, it is a sequence-critical constraint. A summary of the proposed criticality identifier is presented in Table 1 .

For illustration, consider constraint $c_{5}: B \mathrm{SS}(1) \mathrm{D}$ (in Fig. 2) or $c_{5}: B^{-}+1 \leq D^{-}$with $B^{-}=[2 . .5]$ and $D^{-}=$[9] The start times of $B$ and $D$ defined by $c_{5}$ is determined as $B_{5}{ }^{-}=(-\infty . .8]$ and $D_{5}{ }^{-}=[3 . . \infty)$, respectively. Hence, their flexibility times of $B$ and $D$ with respect to constraint $c_{5}$ are determined as:

$$
\begin{aligned}
& f_{B, 5}^{F W}=8-2=6 \\
& f_{B, 5}^{B W}=\infty \\
& f_{D, 5}^{F W}=\infty \\
& f_{D, 5}^{B W}=9-3=6
\end{aligned}
$$

Consequently, the tightening time of $c_{5}$ is determined as $T_{5}=\min (6,6)=6$. Since $T_{5}$ has a non-zero vale and no better makespan is obtained after removing constraint $c_{5}$, this constraint is identified as non-critical in this alternative schedule. Besides, $T_{5}=6$ indicates that constraint $c_{5}$ can still be satisfied with any changes in lag time or activity durations within 6 days. In particular, at the extremities, it is still satisfied if its $m_{5}$ can be increased to $m_{5}=7$ and activity $\mathrm{B}$ is carried out on Day $2\left(B^{-}=2\right)$, 
or if activity $\mathrm{B}$ can be delayed by 4 days $\left(B^{-}=8\right.$ ) and its lag time remains as $m_{5}=1$.

\subsection{Constraint Criticality Identification Procedure}

The general procedure of identifying the criticality of a constraint $C$ consists of four steps as follows:

1. Calculate the flexibility of activities involved following equations (1) and (2).

2. Compute tightening time using equation (3).

3. Classify $C$ as project-critical, activity-critical or non-critical based its tightening time.

4. If $C$ is non-critical, remove $C$ from the constraint collection and reschedule the project. If there exist any change in activity's times or sequence that lead to a similar or shorter duration, then $\mathrm{c}$ is sequence-critical; otherwise, $C$ is non-critical.

\section{Illustrative Case Example}

A case study adopted and modified from Song and Chua (2011) is presented to demonstrate the application of the proposed concept. It based on the construction of the main entrance of a nursing house (shown in Figure 5), which consists of two major tasks: (1) design and construction of the glass work of curtain wall and steel beam, and (2) laying of cable pipe.

Fig. 6 depicts the constraint network considered for this project. The temporal constraints are indicated on the directed arcs. Directed arcs without any indications are assumed to depict $\mathrm{B}(0)$ constraint, which is analogous to the normal precedent constraint (FS0) in CPM/PDM models. Besides normal technological constraints which are represented as $\mathrm{B}(0)$ relationships, the following special constraints have also identified an incorporated into the schedule. Scaffold is needed for the erection of beam and glass. The scaffolding system can be erected only after trench is backfilled $\left(c_{10}\right)$, and removed after the erection of both beam and glass structure is completed. Besides, there is only one crew for the erection of beam and glass; therefore, these processes cannot be carried out concurrently $\left(c_{12}\right)$. Moreover, due to site constraints, the erection of glass can only be started 2 days after the shipping of beam has been finished $\left(c_{7}\right)$.

Due to the existence of a disjunctive constraint, this project has two feasible alternative schedules, and the best one in which Beam Erection is carried out before Glass Erection and total project duration is 27 days is depicted in Fig. 7. The other alternative sequence, where Beam Erection is done after Glass Erection, leads to longer project duration of 29 days. The result of criticality analysis based on this alternative schedule is summarized in Table 2 .

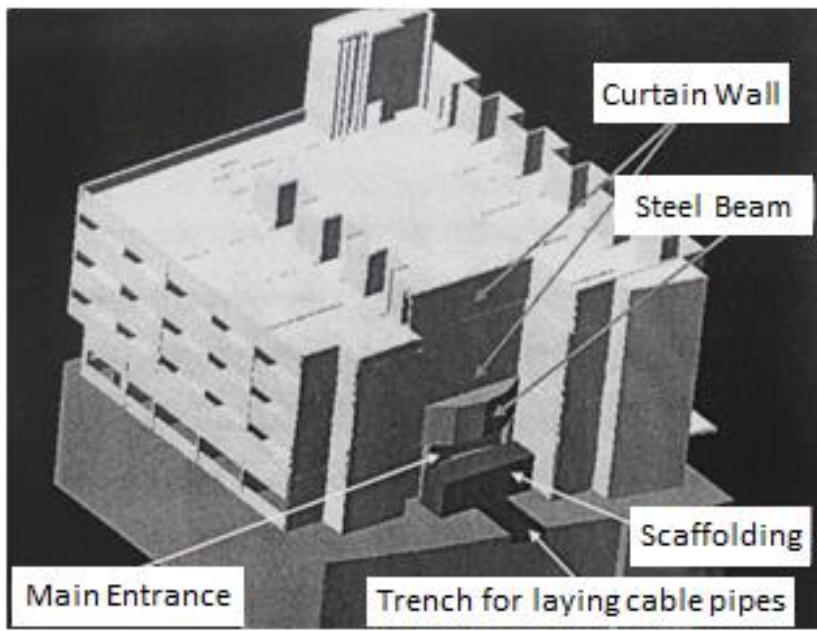

Fig. 5. 3D model of nursing house showing main entrance

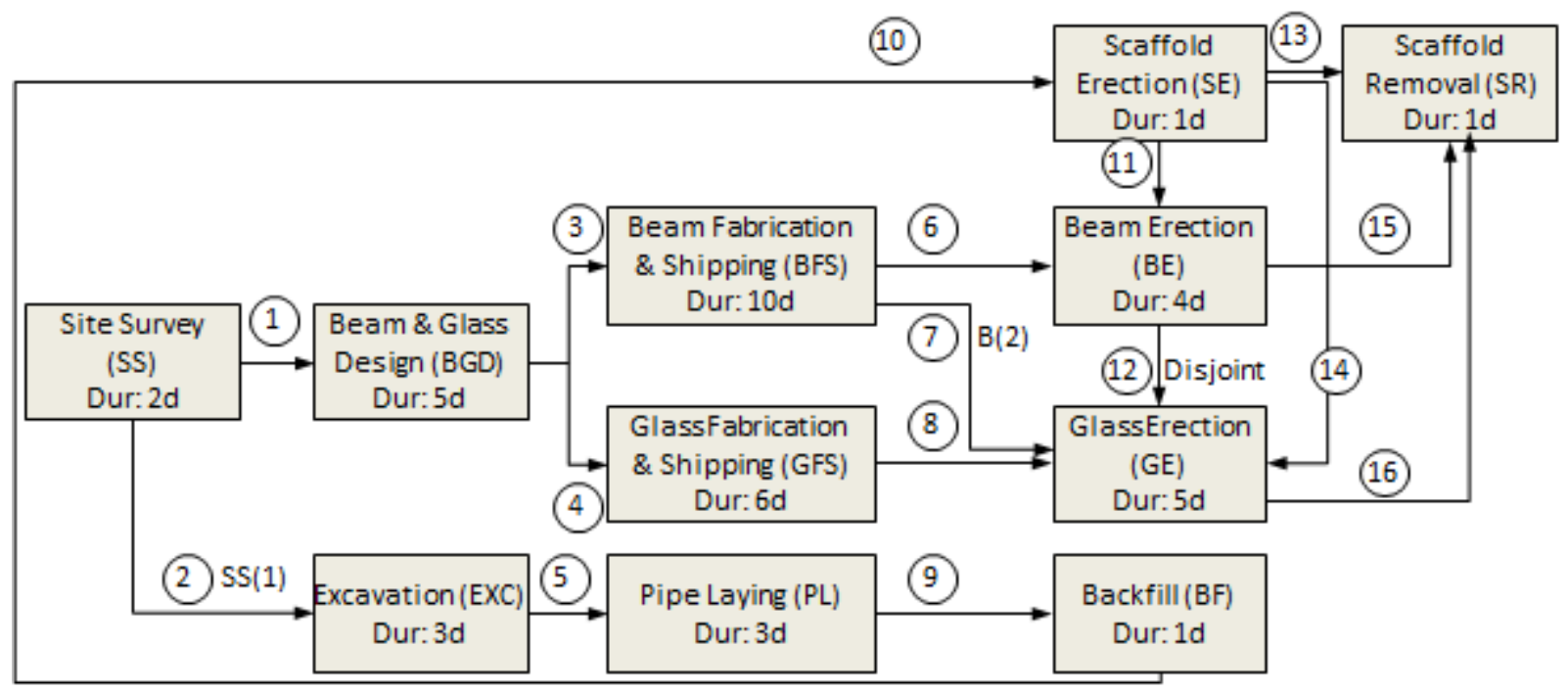

Fig. 6. Constraint network 
Table 2. Tightening time and criticality type of constraints

\begin{tabular}{ccc}
\hline Constraint & $T_{C}$ & Criticality \\
\hline 1 & 0 & project-critical \\
2 & 8 & activity-critical \\
3 & 0 & project-critical \\
4 & 8 & activity-critical \\
5 & 8 & activity-critical \\
6 & 0 & project-critical \\
7 & 2 & sequence-critical \\
8 & 8 & activity-critical \\
9 & 8 & activity-critical \\
10 & 8 & activity-critical \\
11 & 8 & activity-critical \\
12 & 0 & project-critical \\
13 & 17 & non-critical \\
14 & 17 & non-critical \\
15 & 5 & non-critical \\
16 & 0 & project-critical \\
\hline
\end{tabular}

The criticality classification obtained can provide useful information for project planners. Firstly, constraints $c_{1}, c_{3}, c_{6}, c_{12}$, and $c_{16}$ are project-critical. Hence, special management attention needs to be put to these constraints. On the other hand, constraints $c_{2}, c_{4}, c_{5}, c_{8}, c_{9}, c_{10}$, and $c_{11}$ are activity-critical as they govern the start/finish times of non-critical activities. Accordingly, when these constraints are varied within its tightening time, the early/late times of the associated activities and therefore their floats are changed correspondingly while the schedule makespan remain unchanged. It is also interesting to note that constraint $c_{7}$ linking two critical activities BFS and GE has non-zero tightening time could be considered redundant in this current alternative schedule. With $T_{7}=2$, the lag time requirement of $c_{7}$ can be changed from $m_{7}=2$ to $m 7=4$ without leading to any change in the makespan of this schedule. However, when $c_{7}$ is removed, Glass Erection can be carried out before Beam Erection, resulting in a better project completion time of 21 days - an improvement in project duration. Therefore, $c_{7}$ is identified as sequence-critical. Therefore, planners should reconsider the site constraint to see if it can be removed so that better project duration can be obtained.

\section{Constraint Criticality vs. Activity Criticality}

The concept of criticality plays a vital role for schedule management. This concept is traditionally applied from the activity perspective. The major focus is to determine the most crucial or critical activities that have significant impact to the overall schedule. From that, planners could produce a suitable management strategy to reduce the adverse impact of activity changes. Activity criticality is helpful to manage uncertainties at the activity level such as uncertain durations or disruptions. However, this concept could not provide planners with information about which constraints (and which construction requirements in a broader view) could affect an activity, or how an activity could be impact if a certain constraint has variations.

Constraint criticality concept, on the other hand, concentrates on the role of a constraint to activities' times and project duration. It could allow planners to identify which constraints are the key bottlenecks that could have adverse impact to the schedule or which constraints directly govern the sequence among activities. As schedule constraints are generated from construction requirements which commonly vary along the project lifetime, besides critical activities, determining essentially crucial constraints are also necessary for planners to place high priority at the right places, and thus the overall schedule performance could be improved.
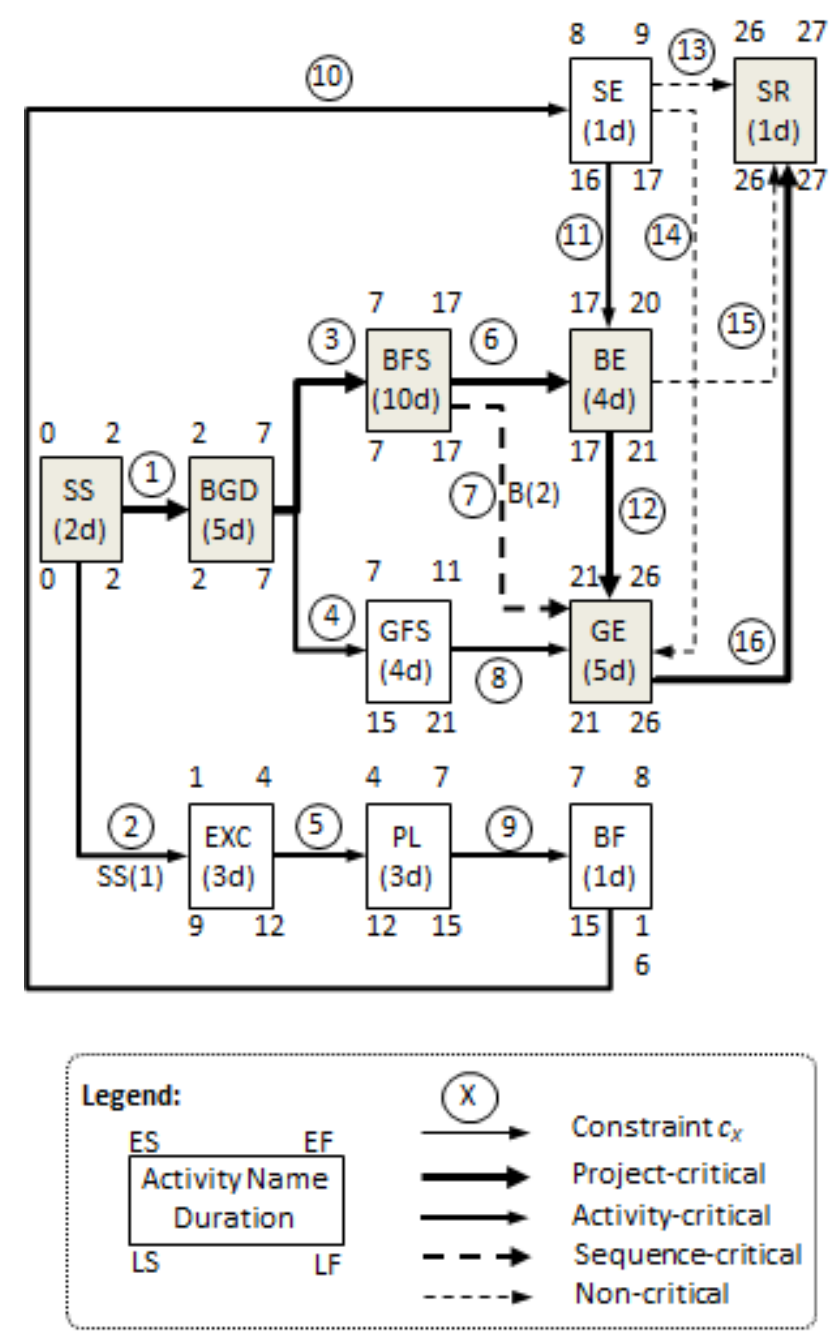

Fig. 7. Best alternative schedule with constraint criticality

\section{Conclusion}

Schedule constraints play a vital role for schedule planning and controlling. They define the temporal relationships and sequences among activities. However, constraints could yield different impact on activities' times and project duration. As constraint criticality differs from one constraint to another, identifying constraint criticality is vital for schedule management.

This paper introduces a classification schema of constraint criticality. In a schedule, a constraint could be project-critical, activity-critical, sequence-critical or noncritical depending on how it could affect activities' start/finishes times and/or project duration. A systematic methodology for identifying constraint criticality is also presented using constraint tightening time as an criticality indicator. Its application was demonstrated via a simple 
schedule example. The result obtained shows that different criticality types can be systematically determined.

A key advantage of the present methodology is that it provides a deeper understanding about the criticality of schedule constraints. As a result, planners could choose appropriate management strategies for each constraint to achieve better project performance. In addition, since schedule constraints are derived from construction requirements which represent project's constraints and concerns in different aspects, this concept of constraint criticality can help further understand the essential of and manage construction requirements at a higher level. Moreover, the present criticality classification provides planners with preliminary understanding on the impact of constraint variations on schedule makespan.

Future extensions to this research include developing new schedule change analysis methodology based on constraint tightening time to analyze the impact of a constraint on schedule makespan when it is varied. In addition, although correlation among schedule constraints possibly exists, the current research did not examine this situation. Future research should attempt to investigate the impact of constraint dependencies to constraint criticality. Such a research would provide more insight knowledge on the nature of constraint criticality.

\section{References}

Al-Otaibi, S., Osmani, M., and Price, A. D. F. (2013). A framework for improving project performance of standard design models in Saudi Arabia. Journal of Engineering, Project, and Production Management 3(2), 85-98.

Allen, J. F. (1984). Towards a general theory of action and time. Artificial Intelligence 23(2), 123-154.

Bowers, J. A. (1995). Criticality in resource constrained networks. The Journal of Operational Research Society 46(1), 80.

Chua, D. K. H. and Shen, L. J. (2005). Key constraints analysis with integrated production scheduler. Journal of Construction Engineering and Management 131(7), 753-764.

Chua, D. K. H. and Yeoh, K. W. (2011). PDM++: Planning framework from a construction requirements perspective. Journal of Construction Engineering and Management 137(4), 266-274.

El-Bibany, H. (1997). Parametric Constraint Management in Planning and Scheduling: Computational Basis. Journal of Construction Engineering and Management 123(3), 348-353.

El-Rayes, K. and Moselhi, O. (2001). Optimizing resource utilization for repetitive construction projects. Journal of Construction Engineering and Management 127(1), 18-27.

Jaafari, A. (1984). Critism of CPM for project planning analysis. Journal of Construction Engineering and Management 110(2), 222-233.

Kelley, J. E. J. (1961). Critical-path planning and scheduling: Mathematical basis. Operations Research 9(3), 296-320.

Lu, M. and Li, H. (2003). Resource-activity critical-path method for construction planning. Journal of Construction Engineering and Management 129(4), 412-420.
Moder, J. J., Phillips, C. R., and Davis, E. W. (1983). Project management with CPM, PERT and precedence diagramming. 3rd edition. Wokingham, Berks., UK, Van Nostrand Reinhold.

Neumann, K. and Zhan, J. (1995). Heuristics for the minimum project-duration problem with minimal and maximal time lags under fixed resource constraints. Journal of Intelligent Manufacturing 6(2), 145-154.

Nguyen, T. Q., Chua, D., and Yeoh, K. (2009). Functional requirement oriented modeling framework for schedule generation. The 6th International Conference on Innovative in Architecture, Engineering \& Construction (AEC), State College, Pennsylvania.

Rahman, S. U. (1998). Theory of constraints: a review of the philosophy and its applications. International Journal of Operations \&amp; Production Management 18(4), 336-355.

Rivera, F. A. and Duran, A. (2004). Critical clouds and critical sets in resource-constrained projects. International Journal of Project Management 22(6), 489-497.

Song, Y. and Chua, D. K. H. (2006). Modeling of functional construction requirements for constructability analysis. Journal of Construction Engineering and Management 132(12), 1314-1326.

Song, Y. and Chua, D. K. H. (2011). Requirement and availability time-window analysis of intermediate function. Journal of Construction Engineering and Management 137(11), 967-975.

Valls, V. and Lino, P. (2001). Criticality analysis in activity-on-node networks with minimal time lags. Annals of Operations Research 102, 17-37.

Wiest, J. D. (1981). Precedence diagramming method: some unsual characteristics and their implications for project managers. Journal of Operations Management 1(3), 121.

Woodworth, B. M. and Shanahan, S. (1988). Identifying the critical sequence in a resource constrained project. International Journal of Project Management 6(2), 89-96.

Yeoh, K. W. (2012). Construction Requirements Driven Planning and Scheduling. Doctoral Dissertation, National Universtity of Singpaore.

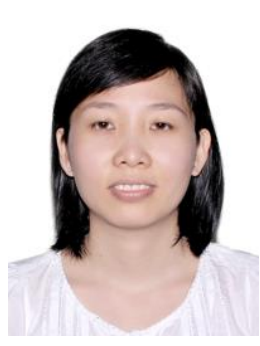

Ms. Nguyen is currently $\mathrm{PhD}$ Candidate in the Department of Civil and Environmental Engineering at National University of Singapore. Her current research focus is on developing concepts and methodologies for automated alternative scheduling, evaluation of schedule alternatives and schedule change management from the perspective of construction requirements. 


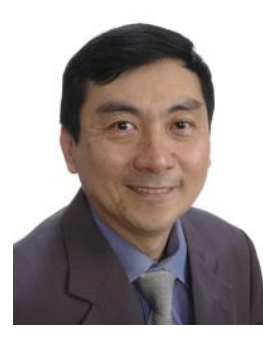

David K. H. Chua is currently an associated professor, Department of Civil and Environmental Engineering at National University of Singapore. $\mathrm{He}$ is the Member of International Group for Lean Construction, Construction Research Council, ASCE, and American Society of Civil Engineers. His research interests include information technology, project management, lean construction and risk management. 